\title{
On Compactness of Embeddings of Fourier-Lebesgue Spaces into Modulation Spaces
}

\author{
Yevgeniy V. Galperin \\ Department of Mathematics, East Stroudsburg University of Pennsylvania, East Stroudsburg, PA, USA \\ Correspondence should be addressed to Yevgeniy V. Galperin; egalperin@po-box.esu.edu
}

Received 7 October 2013; Accepted 18 November 2013

Academic Editor: Remi Léandre

Copyright (c) 2013 Yevgeniy V. Galperin. This is an open access article distributed under the Creative Commons Attribution License, which permits unrestricted use, distribution, and reproduction in any medium, provided the original work is properly cited.

It is shown that, for a certain range of parameters, embeddings of Fourier-Lebesgue $L_{a}^{p} \cap \mathscr{F} L_{b}^{q}$ spaces into modulation spaces $M_{\alpha, \beta}^{r, s}$ are compact.

\section{Introduction}

In [1], Galperin and Gröchenig studied the question of how changing the requirements on smoothness and decay of $f$ and $\widehat{f}$ affects the lower bound in the uncertainty principle. They derived a class of uncertainty principles in the form

$$
\left\|V_{g} f\right\|_{L_{\alpha, \beta}^{r, s}} \leq C\left(\|f\|_{L_{a}^{p}}+\|\hat{f}\|_{L_{b}^{q}}\right)
$$

and partially characterized the range of the parameters $p, q, r$, $s, a, b, \alpha$, and $\beta$ for which (1) holds. Here $\widehat{f}$ is the Fourier transform of $f$ normalized as $\mathscr{F} f(\omega)=\widehat{f}(\omega)=\int_{\mathbb{R}^{d}} f(x) e^{-2 \pi i x \omega} d x$ and the the quantities $\|f\|_{L_{a}^{p}}=\left\|(1+|x|)^{a} f\right\|_{L^{p}}$ and $\|\widehat{f}\|_{L_{b}^{q}}=$ $\left\|(1+|\omega|)^{b} \hat{f}\right\|_{L^{q}}$ are used as measures of the concentration of $f$ in time and frequency, respectively. For a fixed $g \in$ $\delta\left(\mathbb{R}^{d}\right)$, a so-called window function, the STFT of a tempered distribution $f \in \mathcal{S}^{\prime}\left(\mathbb{R}^{d}\right)$ with respect to $g$ is defined by

$$
V_{g} f(x, \omega)=\int_{\mathbb{R}^{d}} f(t) \overline{g(t-x)} e^{-2 \pi i \omega \cdot t} d t=\left\langle f, M_{\omega} T_{x}\right\rangle,
$$

where the translation and modulation operators are defined by $T_{x} g(t)=g(t-x)$ and $M_{\omega} g(t)=e^{2 \pi i \omega t} g(t)$. To measure the joint time-frequency concentration of a function $f$, the mixed weighted Lebesgue norm

$$
\begin{gathered}
\left\|V_{g} f\right\|_{L_{\alpha, \beta}^{r s}}=\left(\int_{\mathbb{R}^{d}}\left(\int_{\mathbb{R}^{d}}\left|V_{g} f(x, \omega)\right|^{r}(1+|x|)^{a r} d x\right)^{s / r}\right. \\
\left.\times(1+|\omega|)^{\beta s} d \omega\right)^{1 / s}
\end{gathered}
$$

is imposed on the short-time Fourier transform (STFT) of $f$. The theory of mixed-norm Lebesgue spaces is developed in [2].

The uncertainty principles of form (1) are equivalent to embeddings of Fourier-Lebesgue spaces into modulation spaces. For a fixed $g \in \delta\left(\mathbb{R}^{d}\right)$, define $M_{\alpha, \beta}^{r, s}$ by the (quasi-)norm $\|f\|_{M_{\alpha, \beta}^{r, s}}=\left\|V_{g} f\right\|_{L_{\alpha, \beta}^{r, s}}$, and then the uncertainty principle (1) is equivalent to the embedding

$$
L_{a}^{p} \cap \mathscr{F} L_{b}^{q} \hookrightarrow M_{\alpha, \beta}^{r, s},
$$

where $\mathscr{F} L_{b}^{q}$ denotes the space of the tempered distributions $f$ whose Fourier transform $\widehat{f}$ is in $L_{b}^{q}$.

In this paper, we show that embeddings (4) are compact. We prove the following theorem.

Theorem 1. Let $0<r, s \leq 2, \alpha, \beta \geq 0$, and $1 \leq p, q \leq \infty$. Suppose that $0<r \leq p \leq \infty$ and $0<s \leq q \leq \infty$. 
If

$$
\begin{aligned}
& \left(\frac{a-\alpha}{d}+\frac{1}{p}-\frac{1}{r}\right)\left(\frac{b-\beta}{d}+\frac{1}{q}-\frac{1}{s}\right) \\
& >\max \left\{\left(\frac{1}{r}-\frac{1}{q^{\prime}}+\frac{\alpha}{d}\right),\left(\frac{1}{r}-\frac{1}{2}+\frac{\alpha}{d}\right)\right\} \\
& \quad \times \max \left\{\left(\frac{1}{s}-\frac{1}{p^{\prime}}+\frac{\beta}{d}\right),\left(\frac{1}{s}-\frac{1}{2}+\frac{\beta}{d}\right)\right\},
\end{aligned}
$$

with all factors being nonnegative, then $L_{a}^{p} \cap \mathscr{F} L_{b}^{q}$ is compactly embedded in $M_{\alpha, \beta}^{r, s}$.

The factors on the left side of (5) are quite natural. When they are both positive, $L_{a}^{p} \hookrightarrow L_{\alpha}^{r}$ and $\mathscr{F} L_{b}^{q} \hookrightarrow \mathscr{F} L_{\beta}^{s}$. Since this is not enough to guarantee that $L_{a}^{p} \cap \mathscr{F} L_{b}^{q} \hookrightarrow M_{\alpha, \beta}^{r, s}$ (because the modulation space norm measures the decay of $f$ in time and in frequency simultaneously, whereas the Lebesgue space norms of $f$ and $\widehat{f}$ treat time and frequency as separate inputs), the term on the right side of (5) indicates the exact measure of additional decay that has to be imposed on $f$ and $\widehat{f}$. However, the strict inequality in (5) implies some excessive decay, which results in tightness of the STFT on sets bounded in $L_{a}^{p} \cap \mathscr{F} L_{b}^{q}$.

It is interesting to compare Lemma 6 and Theorem 1 with the results obtained in [3, Theorem 3.2], which is concerned with compactness of embeddings into modulation space $M_{s}^{p, q}$. Whereas the weights $m_{s}(x, \omega)=(1+|x|+|\omega|)^{s}$ used in [3] assume the same rate of decay of the short-time Fourier transform in the time and frequency variables, the weights used in this paper differentiate between these two rates. Thus, the result proved in [3] is not directly applicable to our case.

Our result relies on the following criterion of compactness in modulation spaces in terms of tightness of the STFT.

Theorem 2 (see [4, Theorem 5]). Assume that $g \in \mathcal{S}\left(\mathbb{R}^{d}\right)$, $0<r, s<\infty$, and $S$ is a closed and bounded subset of $M_{\alpha, \beta}^{r, s}$. Then $S$ is compact in $M_{\alpha, \beta}^{r, s}$ if and only if, for all $\epsilon>0$, there exists a compact set $U \subset \mathbb{R}^{2 d}$, such that

$$
\sup _{f \in S}\left\|\chi_{U^{c}} \cdot V_{g} f\right\|_{L_{\alpha, \beta}^{r, s}}<\epsilon .
$$

Remark. (1) In [4], Theorem 2 was proved in the context of the co-orbit spaces (with more general weight functions) for the case $1 \leq r, s<\infty$. However, the same argument works for $M_{\alpha, \beta}^{r, s}, 0<r, s<\infty$.

(2) For the theory of modulation spaces we refer to [5, Chapter 11-12], [6] and to the original literature [7-9].

(3) It is shown in [1] that condition (5) is optimal. If the inequality is reversed, $L_{a}^{p} \cap \mathscr{F} L_{b}^{q}$ is not embedded in $M_{\alpha, \beta}^{r, s}$.

\section{Definitions and Preliminary Results}

We first provide the necessary definitions and tools. Our notation and definitions are consistent with those in [5].
2.1. Weights and Mixed Norm Spaces. To alleviate notation, we write $\langle x\rangle=1+|x|$. We need the following lemma for weighted mixed norm spaces.

Lemma 3 (Hölder's inequality). Let $p \geq r$ and $q \geq s$. Write $t=r(p / r)^{\prime}=r p /(p-r)$ and $u=s(q / s)^{\prime}=s q /(q-s)$. Then

$$
\|F \cdot H\|_{L_{\alpha, \beta}^{r, s}} \leq\|F\|_{L_{a, b}^{p, q}} \cdot\|H\|_{L_{\alpha-a, \beta-b}^{t, u}}
$$

whenever the right-hand side is finite.

Proof. We write the left-hand side as

$$
\begin{aligned}
\|F \cdot H\|_{L_{\alpha, \beta}^{r, s}}=\left(\int_{\mathbb{R}^{d}}\right. & \left(\int_{\mathbb{R}^{d}}|F(x, \omega)|^{r}\langle x\rangle^{a r}\right. \\
& \left.\times|H(x, \omega)|^{r}\langle x\rangle^{(\alpha-a) r} d x\right)^{s / r} \\
& \left.\times\langle\omega\rangle^{b s}\langle\omega\rangle^{(\beta-b) s} d \omega\right)^{1 / s} .
\end{aligned}
$$

Next apply Hölder's inequality with exponents $p / r$ and $(p / r)^{\prime}$ to the integral in $d x$ and with exponents $q / s$ and $(q / s)^{\prime}$ to the integral in $d \omega$. This yields

$$
\begin{aligned}
\|F \cdot H\|_{L_{\alpha, \beta}^{r, s}}^{r s} & \left(\int_{\mathbb{R}^{d}}\|F(\cdot, \omega)\|_{L_{a}^{p}}^{s}\langle\omega\rangle^{b s}\right. \\
& \left.\cdot\|H(\cdot, \omega)\|_{L_{\alpha-a}^{t}}^{s}\langle\omega\rangle^{(\beta-b) s} d \omega\right)^{1 / s} \\
\leq & \left(\int_{\mathbb{R}^{d}}\|F(\cdot, \omega)\|_{L_{a}^{p}}^{q}\langle\omega\rangle^{b q} d \omega\right)^{1 / q} \\
& \cdot\left(\int_{\mathbb{R}^{d}}\|H(\cdot, \omega)\|_{L_{\alpha-a}^{t}}^{u}\langle\omega\rangle^{(\beta-b) u} d \omega\right)^{1 / u} \\
= & \|F\|_{L_{a, b}^{p, q}} \cdot\|H\|_{L_{\alpha-a, \beta-b}^{t, u}},
\end{aligned}
$$

as desired.

We will also use the following elementary embedding.

Lemma 4. Suppose that $1 \leq r<p \leq \infty$. Then $L_{a}^{p} \hookrightarrow L_{\alpha}^{r}$ if and only if $(a-\alpha) / d+1 / p-1 / r>0$.

The following technical lemma about weighted mixed norms of certain characteristic functions is instrumental for the main embedding result.

Lemma 5 (see [1]). Let $\alpha, \beta \in \mathbb{R}, 0<r, s \leq \infty$, and $\sigma>0$. Define $A_{\sigma}=\left\{(x, \omega) \in \mathbb{R}^{2 d}:|x| \geq|\omega|^{1 / \sigma}\right\}$ and $B_{\sigma}=\mathbb{R}^{2 d} \backslash A_{\sigma}=$ $\left\{(x, \omega) \in \mathbb{R}^{2 d}:|x|<|\omega|^{1 / \sigma}\right\}$.

Then $\chi_{B_{\sigma}} \in L_{\alpha, \beta}^{r, s}$ provided that

$$
\frac{1}{\sigma} \max \left\{\frac{\alpha}{d}+\frac{1}{r}, 0\right\}+\frac{\beta}{d}+\frac{1}{s}<0 .
$$


Furthermore, $\chi_{A_{\sigma}} \in L_{\alpha, \beta}^{r, s}$ provided that

$$
\begin{gathered}
\frac{\alpha}{d}+\frac{1}{r}<0, \\
\frac{1}{\sigma}\left(\frac{\alpha}{d}+\frac{1}{r}\right)+\frac{\beta}{d}+\frac{1}{s}<0 .
\end{gathered}
$$

\section{Proof of the Main Result}

In order to prove Theorem 1, we first establish compactness of certain embeddings between modulation spaces.

Lemma 6. Assume that $0<r, s<\infty, \alpha, \beta \geq 0$, and $p, q^{\prime} \in$ $\left[r, s^{\prime}\right]$. If

$$
\begin{gathered}
\left(\frac{a-\alpha}{d}+\frac{1}{p}-\frac{1}{r}\right)\left(\frac{b-\beta}{d}+\frac{1}{q}-\frac{1}{s}\right) \\
>\left(\frac{\alpha}{d}-\frac{1}{q^{\prime}}+\frac{1}{r}\right)\left(\frac{\beta}{d}-\frac{1}{p^{\prime}}+\frac{1}{s}\right)
\end{gathered}
$$

with all factors nonnegative, then $M_{a, 0}^{p, p^{\prime}} \cap M_{0, b}^{q^{\prime}, q}$ is compactly embedded in $M_{\alpha, \beta}^{r, s}$.

Proof. We split the time-frequency plane into the two regions $A_{\sigma}=\left\{(x, \omega) \in \mathbb{R}^{2 d}:|x| \geq|\omega|^{1 / \sigma}\right\}$ and $B_{\sigma}=\mathbb{R}^{2 d} \backslash A_{\sigma}$ for some $\sigma>0$ to be determined later, and we estimate the modulation space (quasi-)norm of $f$ accordingly by

$$
\begin{aligned}
\|f\|_{M_{\alpha, \beta}^{r, s}} & =\left\|V_{g} f\right\|_{L_{\alpha, \beta}^{r, s}} \\
& \leq C\left(\left\|V_{g} f \cdot \chi_{A_{\sigma}}\right\|_{L_{\alpha, \beta}^{r, s}}+\left\|V_{g} f \cdot \chi_{B_{\sigma}}\right\|_{L_{\alpha, \beta}^{r, s}}\right) .
\end{aligned}
$$

We then apply Hölder's inequality (Lemma 3 ) to each term and use Lemma 5. Writing $t=r(p / r)^{\prime}=r p /(p-r)$ and $u=$ $s\left(p^{\prime} / s\right)^{\prime}=s p^{\prime} /\left(p^{\prime}-s\right)$, we obtain that

$$
\begin{aligned}
\left\|V_{g} f \cdot \chi_{A_{\sigma}}\right\|_{L_{\alpha, \beta}^{r, s}} & \leq\left\|V_{g} f\right\|_{L_{a, 0}^{p, p^{\prime}}} \cdot\left\|\chi_{A_{\sigma}}\right\|_{L_{\alpha-a, \beta}^{t, u}} \\
& =\|f\|_{M_{a, 0}^{p, p^{\prime}}} \cdot\left\|\chi_{A_{\sigma}}\right\|_{L_{\alpha-a, \beta}^{t, u} \cdot} \cdot
\end{aligned}
$$

Lemma 5 implies that $\chi_{A_{\sigma}} \in L_{\alpha-a, \beta}^{t, u}$, whenever

$$
\begin{gathered}
\frac{\alpha-a}{d}+\frac{1}{t}=\frac{\alpha-a}{d}+\frac{1}{r}-\frac{1}{p}<0, \\
\frac{1}{\sigma}\left(\frac{\alpha-a}{d}+\frac{1}{t}\right)+\frac{\beta}{d}+\frac{1}{u}<0 .
\end{gathered}
$$

Equivalently,

$$
\frac{a-\alpha}{d}+\frac{1}{p}-\frac{1}{r}>\sigma\left(\frac{\beta}{d}-\frac{1}{p^{\prime}}+\frac{1}{s}\right) \geq 0 .
$$

Similarly, we obtain for the second term that

$$
\left\|V_{g} f \cdot \chi_{B_{\sigma}}\right\|_{L_{\alpha, \beta}^{r, s}} \leq\left\|V_{g} f\right\|_{L_{0, b}^{q^{\prime}, q}} \cdot\left\|\chi_{B_{\sigma}}\right\|_{L_{\alpha, \beta-b}^{\tilde{t}, \tilde{u}}},
$$

where $\tilde{t}=r\left(q^{\prime} / r\right)^{\prime}=r q^{\prime} /\left(q^{\prime}-r\right)$ and $\tilde{u}=s(q / s)^{\prime}=s q /(q-s)$. By Lemma 5 we have $\chi_{B_{\sigma}} \in L_{\alpha, \beta-b}^{\widetilde{t}, \widetilde{u}}$ provided that

$$
\frac{1}{\sigma} \max \left\{\frac{\alpha}{d}+\frac{1}{\widetilde{t}}, 0\right\}+\frac{\beta-b}{d}+\frac{1}{\widetilde{u}}<0,
$$

or equivalently,

$$
\begin{aligned}
\frac{b-\beta}{d}+\frac{1}{q}-\frac{1}{s} & >\frac{1}{\sigma} \max \left\{\frac{\alpha}{d}-\frac{1}{q^{\prime}}+\frac{1}{r}, 0\right\} \\
& =\frac{1}{\sigma}\left(\frac{\alpha}{d}-\frac{1}{q^{\prime}}+\frac{1}{r}\right) \geq 0 .
\end{aligned}
$$

Finally, if (12) holds, then there exists $\sigma>0$ so that both (16) and (19) and all factors are positive. Hence, $\chi_{A_{\sigma}} \in L_{\alpha-a, \beta}^{t, u}$ and $\chi_{B_{\sigma}} \in L_{\alpha, \beta-b}^{\tilde{t}, \tilde{u}}$. It follows that, for a given $\epsilon>0$, there exist compact sets $U_{1}, U_{2} \subset \mathbb{R}^{2 d}$, such that

$$
\left\|\chi_{A_{\sigma}} \cdot \chi_{U_{1}^{c}}\right\|_{L_{\alpha-a, \beta}^{t, u}}<\frac{\epsilon}{2 C}, \quad\left\|\chi_{B_{\sigma}} \cdot \chi_{U_{2}^{c}}\right\|_{L_{\alpha, \beta-b}^{\tilde{\tau}, \tilde{u}}}<\frac{\epsilon}{2 C} .
$$

Define $U=U_{1} \cup U_{2}$. The combination of (14), (17), and (20) yields that

$$
\sup _{f \in \operatorname{Ball}\left(M_{a, 0}^{p, p^{\prime}} \cap M_{0, b}^{q^{\prime}, q}\right)}\left\|\chi_{U^{c}} \cdot V_{g} f\right\|_{L_{\alpha, \beta}^{r, s}}<\epsilon .
$$

Therefore, by Theorem 2, the embedding $M_{a, 0}^{p, p^{\prime}} \cap M_{0, b}^{q^{\prime}, q} \hookrightarrow$ $M_{\alpha, \beta}^{r, s}$ is compact.

The next lemma relates weighted $L^{p}$ spaces to modulation spaces and can be considered a version of the HausdorffYoung inequality for the STFT. For more general inequalities see [10, Section 4.2.1].

Lemma 7 (see $[1,11])$. Suppose that $1 \leq p \leq 2$. Then

(a) $L_{a}^{p} \hookrightarrow M_{a, 0}^{p, p^{\prime}}$ and

$$
\left\|V_{g} f\right\|_{L_{a, 0}^{p, p^{\prime}}} \leq\|f\|_{L_{a}^{p}}\|g\|_{L_{|a|}^{p}},
$$

where $C=C(p, a)$ is independent of $f$ and $g$,

(b) $\mathscr{F} L_{a}^{p} \hookrightarrow M_{0, a}^{p^{\prime}, p}$ and

$$
\left\|V_{g} f\right\|_{L_{0, a}^{p^{\prime}, p}} \leq\|\hat{f}\|_{L_{a}^{p}}\|\hat{g}\|_{L_{|a|}^{p}}
$$

where $C=C(p, a)$ is independent of $f$ and $g$.

The combination of Lemmas 6 and 7 leads to Theorem 1.

Proof of Theorem 1. Following the proof of the main result in [1], we distinguish several cases.

Case 1. If $r \leq p \leq 2$ and $s \leq q \leq 2$, then $L_{a}^{p} \hookrightarrow M_{a, 0}^{p, p^{\prime}}$ and $\mathscr{F} L_{b}^{q} \hookrightarrow M_{0, b}^{q^{\prime}, q}$ by Lemma 7. As a consequence of (5), Lemma 6 is applicable and thus $M_{a, 0}^{p, p^{\prime}} \cap M_{0, b}^{q^{\prime}, q}$ is compactly 
embedded in $M_{\alpha, \beta}^{r, s}$. Combining the above, we obtain that $L_{a}^{p} \cap \mathscr{F} L_{b}^{q}$ is compactly embedded in $L_{\alpha, \beta}^{r, s}$.

Case 2. $p>2, s \leq q \leq 2$.

By continuity there exists $c>0$ such that

$$
\begin{aligned}
& \left(\frac{a-\alpha}{d}+\frac{1}{p}-\frac{1}{r}\right)\left(\frac{b-\beta}{d}+\frac{1}{q}-\frac{1}{s}\right) \\
& >\left(\frac{c-\alpha}{d}+\frac{1}{2}-\frac{1}{r}\right)\left(\frac{b-\beta}{d}+\frac{1}{q}-\frac{1}{s}\right) \\
& >\left(\frac{1}{r}-\frac{1}{q^{\prime}}+\frac{\alpha}{d}\right)\left(\frac{1}{s}-\frac{1}{2}+\frac{\beta}{d}\right) .
\end{aligned}
$$

The first inequality in (24) implies that $(a-c) / d+1 / p-$ $1 / 2>0$ and thus $L_{a}^{p} \hookrightarrow L_{c}^{2}$ by Lemma 4 . In view of (5) Case 1 is now applicable with $L_{c}^{2}$ instead of $L_{a}^{p}$, and we obtain the compact embedding

$$
L_{a}^{p} \cap \mathscr{F} L_{b}^{q} \hookrightarrow L_{c}^{2} \cap \mathscr{F} L_{b}^{q} \hookrightarrow M_{\alpha, \beta}^{r, s} .
$$

Case 3. $q>2, r \leq p \leq 2$ is similar.

\section{Case 4. $p, q>2$.}

By continuity we may choose $c>0$ and $\gamma>0$, so that the inequalities $(a-c) / d+1 / p-1 / 2>0,(b-\gamma) / d+1 / q-1 / 2>0$, and

$$
\begin{aligned}
& \left(\frac{a-\alpha}{d}+\frac{1}{p}-\frac{1}{r}\right)\left(\frac{b-\beta}{d}+\frac{1}{q}-\frac{1}{s}\right) \\
& >\left(\frac{c-\alpha}{d}+\frac{1}{2}-\frac{1}{r}\right)\left(\frac{\gamma-\beta}{d}+\frac{1}{2}-\frac{1}{s}\right) \\
& >\left(\frac{1}{r}-\frac{1}{2}+\frac{\alpha}{d}\right)\left(\frac{1}{s}-\frac{1}{2}+\frac{\beta}{d}\right)
\end{aligned}
$$

hold simultaneously. It follows that $L_{a}^{p} \hookrightarrow L_{c}^{2}$ and $\mathscr{F} L_{b}^{q} \hookrightarrow$ $\mathscr{F} L_{\gamma}^{2}$ by Lemma 4 and that $L_{c}^{2} \cap \mathscr{F} L_{\gamma}^{2}$ is compactly embedded in $M_{\alpha, \beta}^{r, s}$ by Lemmas 6 and 7. Therefore $L_{a}^{p} \cap \mathscr{F} L_{b}^{q}$ is compactly embedded in $M_{\alpha, \beta}^{r, s}$, as desired. The theorem is proved completely.

\section{Acknowledgments}

The author would like to thank K. Gröchenig and H. G. Feichtinger for inspiring discussions and important suggestions.

\section{References}

[1] Y. V. Galperin and K. Gröchenig, "Uncertainty principles as embeddings of modulation spaces," Journal of Mathematical Analysis and Applications, vol. 274, no. 1, pp. 181-202, 2002.

[2] A. Benedek and R. Panzone, "The space $L^{p}$, with mixed norm," Duke Mathematical Journal, vol. 28, pp. 301-324, 1961.

[3] A. Hinrichs, I. Piotrowska, and M. Piotrowski, "On the degree of compactness of embeddings between weighted modulation spaces," Journal of Function Spaces and Applications, vol. 6, no. 3, pp. 303-317, 2008.
[4] M. Dörfler, H. G. Feichtinger, and K. Gröchenig, "Compactness criteria in function spaces," Colloquium Mathematicum, vol. 94, no. 1, pp. 37-50, 2002.

[5] K. Gröchenig, Foundations of time-frequency analysis, Applied and Numerical Harmonic Analysis, Birkhäuser, Boston, Mass, USA, 2001.

[6] Y. V. Galperin and S. Samarah, "Time-frequency analysis on modulation spaces $M_{m}^{p, q}, 0<p, q \leq \infty$," Applied and Computational Harmonic Analysis, vol. 16, no. 1, pp. 1-18, 2004.

[7] H. G. Feichtinger, "Modulation spaces on locally compact abelian groups,” Technical Report, University of Vienna, 1983.

[8] H. G. Feichtinger, "Atomic characterizations of modulation spaces through Gabor-type representations," The Rocky Mountain Journal of Mathematics, vol. 19, no. 1, pp. 113-125, 1989.

[9] H. G. Feichtinger and K. Gröchenig, "Gabor wavelets and the Heisenberg group: Gabor expansions and short time Fourier transform from the group theoretical point of view," in Wavelets, vol. 2 of Wavelet Analysis and its Applications, pp. 359-397, Academic Press, Boston, Mass, USA, 1992.

[10] J. A. Hogan and J. D. Lakey, "Embeddings and uncertainty principles for generalized modulation spaces," in Modern Sampling Theory, Applied and Numerical Harmonic Analysis, pp. 73-105, Birkhäuser, Boston, Mass, USA, 2001.

[11] K. Gröchenig, "An uncertainty principle related to the Poisson summation formula," Studia Mathematica, vol. 121, no. 1, pp. 87$104,1996$. 


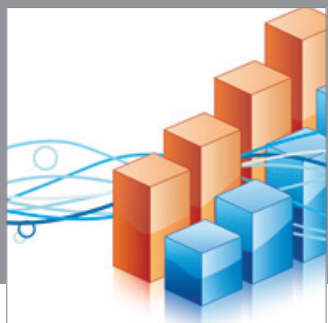

Advances in

Operations Research

mansans

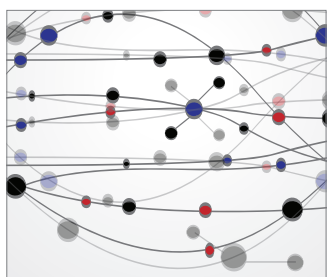

The Scientific World Journal
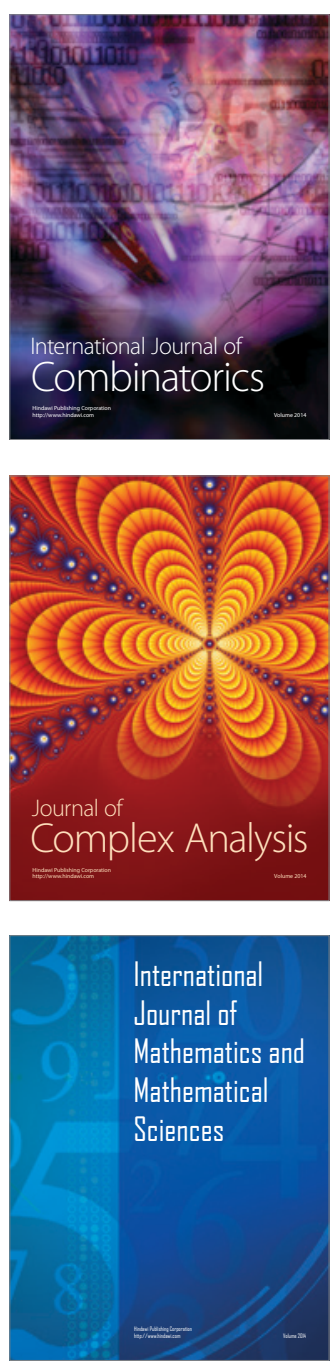
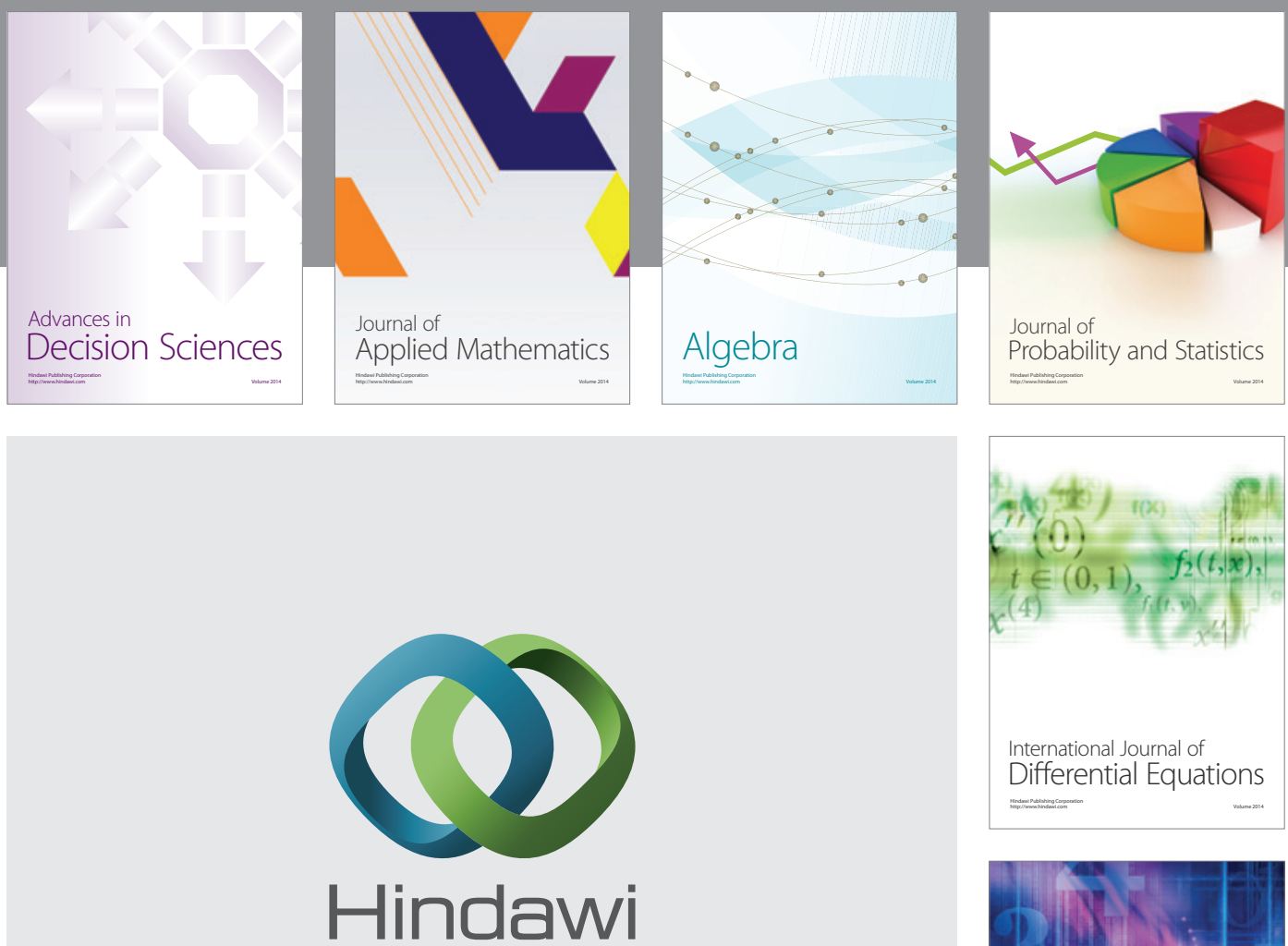

Submit your manuscripts at http://www.hindawi.com
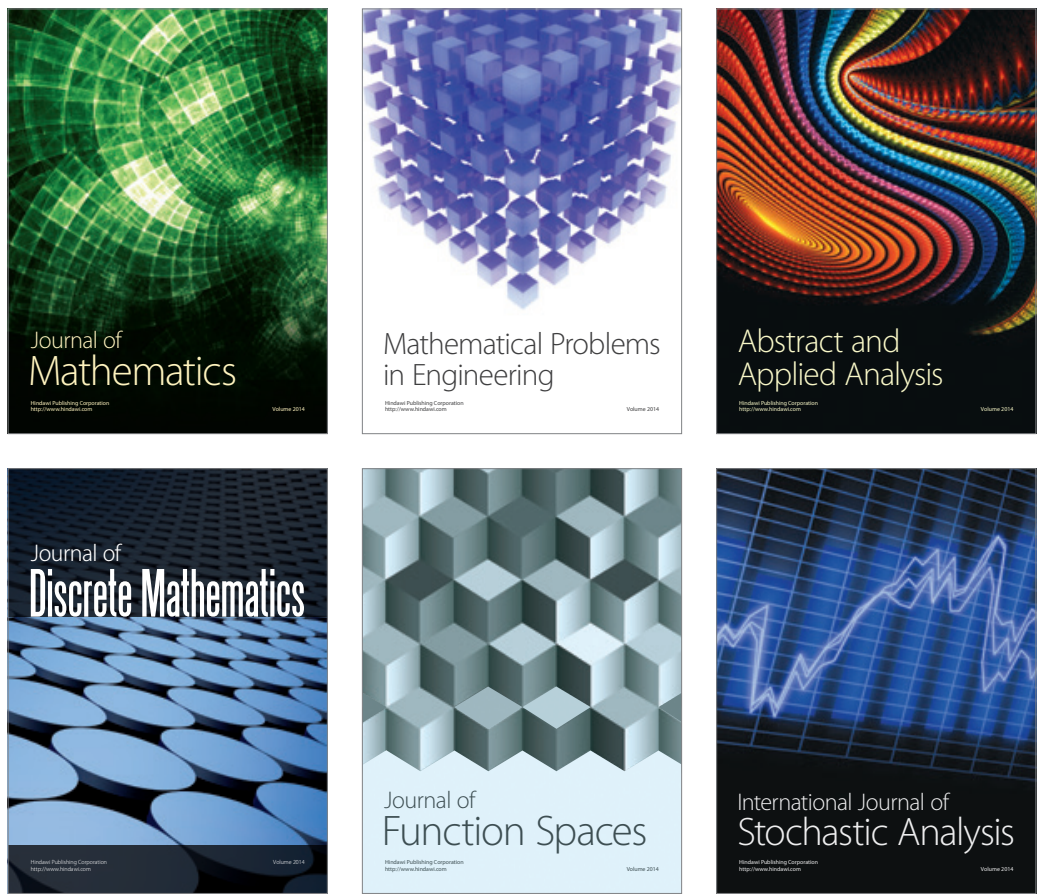

Journal of

Function Spaces

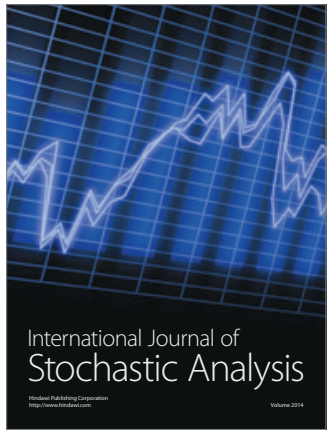

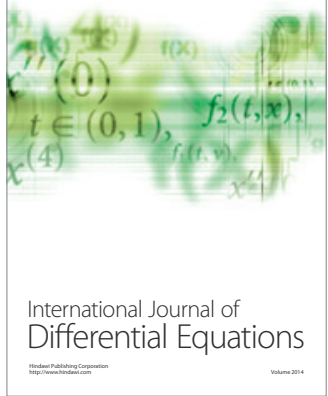
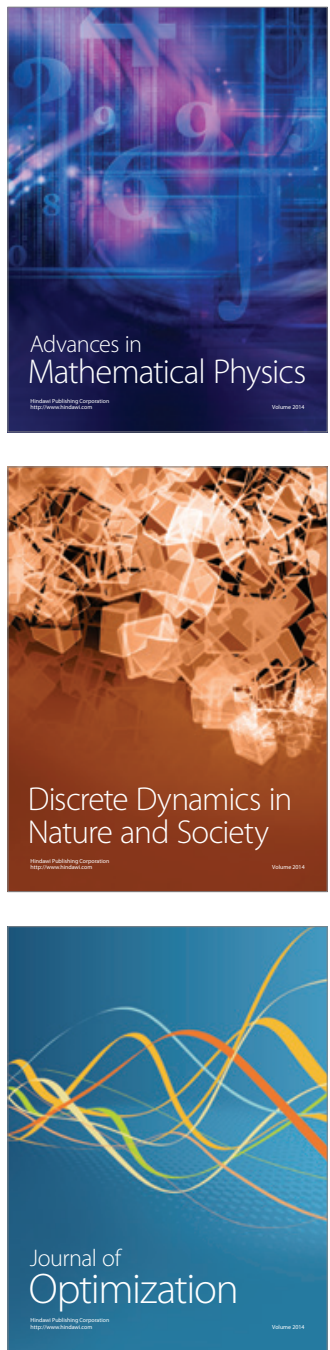